\title{
Biofluid-Based Circulating Tumor Molecules as Diagnostic Tools for Use in Personalized Medicine
}

Yutian Li and EI Mustapha Bahassi*

Department of Internal Medicine; Division of Hematology/Oncology; Cincinnati OH, USA

\begin{abstract}
Circulating Tumor Cells (CTCs), circulating tumor DNA (ctDNA) and Extracellular Vesicles (ECVs) hold the key to understanding the biology of tumor metastasis and provide a biomarker to noninvasively measure the evolution of tumor genotypes during treatment and disease progression. CTCs can be detected in blood from patients with metastatic and primary carcinomas. Over the past decade, the development of immune-magnetic platforms has permitted accurate enumeration of CTCs at extremely low frequencies. In several case reports, the presence of CTCs has been associated with shortened survival times. Circulating-mutant DNA in blood is believed to originate from apoptotic and necrotic cells of the primary tumor, which discharge their DNA early during tumorigenesis. It is conceivable that cell-free tumor-specific DNA could also be related to the rate of turnover of CTCs. Extracellular Vesicles (ECVs) are nano-sized vesicles released by all cells in vitro as well as in vivo. Their role has been implicated mainly in cell-cell communication, but also in disease biomarkers and more recently in gene delivery. They represent a snapshot of the cell status at the moment of release and carry bio-reactive macromolecules such as nucleic acids, proteins, and lipids. Improvements in technologies to isolate purer CTCs, ECVs and ctDNAs will help in a better characterization and enable a broad range of clinical applications, including early detection of disease and the discovery of personalized biomarkers to predict treatment responses and disease progression. This review highlights the progress made in the development of noninvasive biomarkers using CTCs, ctDNA and ECVs.
\end{abstract}

Keywords: Biomarker, Circulating tumor cell, Circulating tumor DNA, Exosome, microRNA, Tumor metastasis, Diagnostic tools, Personalized medicine

\section{Introduction}

If biomarkers for early detection could be discovered, the impact on public health would be staggering. Discovering such biomarkers for cancer, traumatic brain injury, viral and bacterial infection, heart disease, etc. would help the number of deaths drop dramatically. Such biomarkers that could monitor therapeutic effectiveness would enable the correct treatment to be recognized sooner in cases where the best course of treatment is not obvious.

In cancer, providing an early and accurate diagnosis represents one of the major challenges in the battle against tumors. Currently, a tissue biopsy is generally required in order for the doctors to formulate a correct diagnosis of the type of cancer and its progression status. However, for some tumor types, biopsies are invasive procedures with potential damaging side effects that, in extreme cases, could be life threatening. It is therefore appealing to search for minimally invasive biomarkers to detect and monitor disease progression at multiple time points during the course of treatment. Promising alternatives to biopsies that hold the potential to revolutionize the cancer diagnosis field comes from studies on the communication between cancer cells and their microenvironment. Among such noninvasive biomarkers, exosomes, circulating tumor cells, and circulating tumor DNA are the three prominent examples for cancer prognosis and diagnosis (Figure 1). The appropriate use of these biomarkers is still under development and needs to be refined and validated. This mini-review article focuses on the challenges and opportunities of using these biomarkers and the isolation and characterization methods for each of them.

\section{Exosomes}

\section{Exosomes biogenesis}

Transporting biological materials across membranes is critical to maintain normal cell homeostasis, it's composed of active, passive,

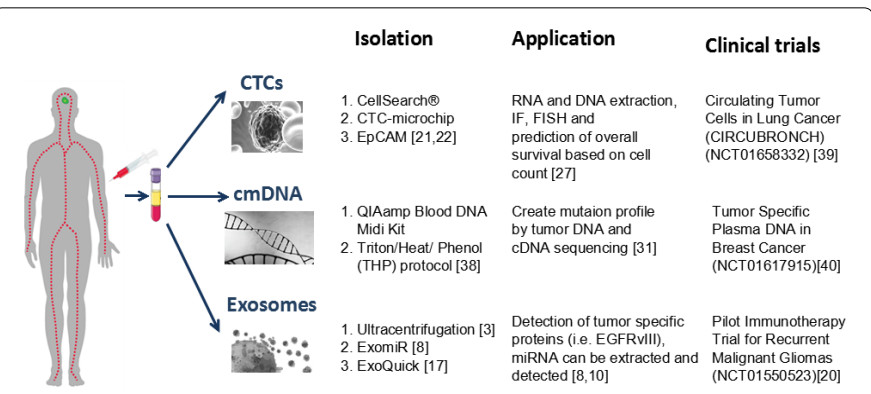

Figure 1: Overview of biofluid-based circulating tumor molecules. For each tumor-derived circulating biomarker, several commonly accepted isolation methods are listed with their application; and ongoing clinical trials are also included to validate their applicability.

export through microparticles, and exosomes that collectively maintain proper compartmentalization of important micro- and macromolecules. Exosomes are microvesicles shed from many different cell types under both normal and pathological conditions, they are also found to occur naturally in body fluids such as blood, urine, break milk, and urine. Their diameters range from $30 \mathrm{~nm}$ to $100 \mathrm{~nm}$. These exosomes can be formed through inward budding of endosomal membranes, leading to the formation of intracellular Multi-Vascular

*Corresponding author: El Mustapha Bahassi, Department of Internal Medicine, Division of Hematology/Oncology, 231, Albert Sabin Way, Cincinnati, OH, 45267 0508, USA, Tel: 513-558 6116; E-mail: bhassiem@uc.edu

Received November 04, 2013; Accepted December 09, 2013; Published December 13, 2013

Citation: Li Y, Mustapha Bahassi El (2013) Biofluid-Based Circulating Tumor Molecules as Diagnostic Tools for Use in Personalized Medicine. J Mol Biomark Diagn 4: 157. doi:10.4172/2155-9929.1000157

Copyright: $\odot 2013 \mathrm{Li} \mathrm{Y}$, et al. This is an open-access article distributed under the terms of the Creative Commons Attribution License, which permits unrestricted use, distribution, and reproduction in any medium, provided the original author and source are credited 


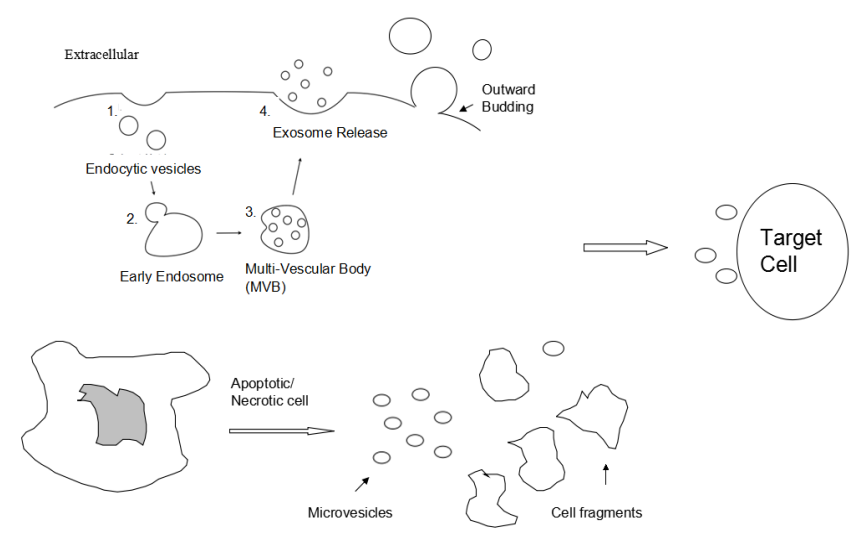

Figure 2: Exosome biogenesis. Inward budding of endosomal membrane results in the formation of intracellular Multi-Vescular Bodies (MVBs), which will fuse with the plasma membrane and release the exosomes to the extracellular area. Exosomes can also be shed by outward budding of the plasma membrane or during cell apoptosis and necrosis, then entering the extracellular area. Exosomes then interact with target cells either by releasing its content (proteins, DNA, RNA, or even miRNA) or fusing with the plasma membrane $[1,41]$.

Bodies (MVB) that will fuse with the plasma membrane, releasing the exosome to the extracellular area. Exosomes can also be shed directly by outward budding of the plasma membrane, then entering the extracellular compartment (Figure 2) [1]. Exosomes were first discovered in maturing mammalian reticulocytes [2]. Originally, they were thought to function as a way for reticulocytes to eliminate the transferrin receptor while maturing [3]. Now exosomes are conceived to play important roles in inter- and intracellular communication [4]. Exosomes consist of a lipid bilayer membrane surrounding a small cytosol lacking any of the cellular organelles, and they contain virtually every type of protein, RNAs or even miRNAs, biological products, viruses [2]. However, the content and biological functions of exosomes depend on the cells of origin. For example, most exosomes excreted by tumor cells suppress immune responses and promote tumor growth and invasiveness, but from Dendritic Cells (DC), exosomes contain co-simulatory proteins necessary for T-cell activation, and exosomes derived from B-cells have potent immunostimulatory and antitumor effect in vivo and have been used as antitumor vaccines $[5,6]$; moreover, brain tumor exosomes could escape the blood-brain barrier and have been shown to express the mutated tumor antigen EGFRvIII and the putatively immunosuppressive cytokine TGF- $\beta$ [7]

\section{Exosome-excreted biological products}

Despite of various origins, exosomes have an evolutionaryconserved common set of proteins, they all contain membrane transport and fusion proteins (GTPases, Annexins, flotillin), tetraspannins (CD9, CD63, CD81, CD82), heat shock proteins (Hsc70, Hsp 90), proteins involved in MVB biogenesis (Alix, TSG101), as well as lipid-related proteins and phospholipases [8]. Depending on the context, proteins and transcription factors carried within exosomes can be tumor promoters or tumor suppressors [2]. A proteomic study investigating exosomes production by $\mathrm{p} 53$, the authors demonstrated that $\mathrm{p} 53$ promotes exosomes production leading to secretion of numerous p53 target cells in the extracellular environment [2]; moreover, the authors also proved that $\mathrm{p} 53$ regulates transcription of many important genes such as TSAP6 and CHMP4C, which enhance exosome production and endosomal clearance of EGFR receptor from the plasma membrane. This mechanism has been shown to help maintain cell growth, restrain cell division, and has a major implication in adjacent cell communication and immune activation [9]. However, much more still needs to be learned about the role of exosomes in p53 pathway, and about the exosomal regulation of p53 and its targets which needs to be considered in the design of any therapeutic strategy against the p53.

Another major component found in circulating exosomes is miRNAs, which are very similar to that found in their originating cancer cells as has been demonstrated [10]. MiRNAs regulate genes by weakly binding to 3'UTR of their target mRNA and therefore, inactivating gene expression [11]. Multiple genes, either within a single cell or in adjacent cells within the microenvironment, can be influenced by miRNAs; indeed, miRNAs can affect distant cells via exosome "carriers" [2]. Exosome-mediated miRNA transfer was first shown in a study that transferred mouse exosomal RNA to human mast cells resulting in the expression of mouse proteins in human cells. Another study monitored the miRNA level in exosomes derived from human breast milk for several month of lactation, the results showed that certain miRNAs, especially miR-181a and miR-155, which play important role in immune system, were present at high levels in the first 6 months, but significantly dropped later, they concluded that miRNAs contained within exosomes from breast milk regulate the development of infant's immune system [8]. In relation to tumors, one study showed that exosome-mediated miRNAs enhanced the invasive potential of breast cancer cell lines [2]. Overall, exosomes play an important role in miRNA regulation and should not be ignored when studying miRNA targets or designing miRNA targeted therapeutic strategies.

Other studies have shown that part of the exosomes' physiological role is their ability to modify the microenvironment through their cargo, which may support tumor survival and metastasis [2]. Very recently, exosomes were demonstrated to reflect the hypoxic status of glioma cells. In this study, activation of vascular cells during tumor formation was observed along with enhanced exosome secretion in a hypoxia dependent manner [2]. In a breast cancer model, hypoxia-mediated activation of HIF-1 a promotes the release of exosomes resulting in a more aggressive cell phenotype [12]. Tumor-derived exosomes may also help create an immunosuppressive microenvironment by inducing apoptosis and impairing the function of effector T cells and NK cells, enhancing angiogenesis, remodeling stromal cells, and promoting extracellular matrix degradation [13]. All these process contribute to the establishment of a pre-metastatic niche.

Exosomes may also play an important role in understanding tumor metastasis. Tumor metastasis causes the most cancer death, but the mechanisms underlying its etiology remain ill understood [2] Exosomes are known to function as escape routes for proteins and miRNAs (might be tumor promoters for some metastatic pathways) from one cell (site of origin) to distant locations. It has been shown that exosomes released from renal cells can promote angiogenesis in lung cancer ascites [14]. As fluorescent exosome-labeling techniques were developed, better understanding of exosome's interaction with angiogenesis, endothelial cells, and metastasis promoters was achieved. Using this technique, melanoma exosomes were observed to interact with and influence endothelial cell morphology [15]. This technique also revealed many examples where tumor exosomes elicited paracrine endothelial signal; and therefore contributing to metastatic spread of tumors, and several other studies have also revealed that tumor exosomes can modulate pre-metastatic cells predominantly through transferred miRNAs and transform them to become tumor cells [2].

Finally, exosomes play an important role in modulating various pathways which lead to development of cancer radio and drug 
resistance. The development of resistance is multifactorial and includes presence of resistant cancer stem-like cells with enhanced plasticity and invasiveness [16], Epithelial-To-Mesenchymal Transition (EMT) phenotype promoted by exosome-released factors, exosome-mediated export of biological material that induces a microenvironment favorable for resistance; resistance can also occur as a result of promoted immune escape mechanisms and fibroblast-like cell formation that causes Desmoplastic Reaction (DR) [2]. As mentioned above, miRNA transported by exosomes can influence multiple signaling pathways that collectively promote resistant phenotype of most cancers. Drug resistance has become an area of intense research and large scale proteomic analysis of tumor-derived exosomes will provide deeper insight of the contents and the potential on clinical application in therapy regimens.

\section{Isolation and characterization of exosomes}

Isolation of exosomes using ultracentrifugation is commonly accepted, oftentimes, this protocol is used in combination with sucrose density gradients or sucrose cushion to float the relatively low-density exosomes, and it could provide a high enrichment of exosomes. However, this protocol is relatively cumbersome and may be complicated by other microvesicles or macromolecular complexes that have similar sizes [3].

Nevertheless, there are alternative exosome isolation routes based on sizes other than ultracentrifugation. A isolation kit (ExomiR) developed by Bioo Scientific could essentially remove all cells, platelets, cellular debris on one microfilter and captures all vesicles bigger than $30 \mathrm{~nm}$ on a second microfilter using positive pressure to drive the fluid [8]. Exosomes might also be precipitated based on differential solubility in alternative solvents, and the precipitate can then be isolated using either low-speed centrifugation or filtration. Recently, a reagent called ExoQuick was released by System Biosciences. It is claimed that ExoQuick can be added to serum, conditioned media, or urine to precipitate the exosomes. The isolation process is very fast and straightforward, but it lacked specificity toward exosomes and the generated pellet is rather difficult to resuspend. However, another study compared the currently utilized methods for purifying exosomes concluded that, while each method purifies exosomal material, exosomes produced by ExoQuick precipitation produces exosomal RNA and protein with greater purity and quantity than chromatography, ultracentrifugation, and DynaBeads [17].

\section{Exosome-based immunotherapy}

There were several Phase I clinical studies with exosomes as immunovaccines. The first one was conducted with autologous Dendritic Cell (DC) Derived-Exosomes (DEX) as vaccine administered intradermally $\left(1 / 10^{\text {th }}\right)$ and subcutaneously $\left(9 / 10^{\text {th }}\right)$ in a weekly interval. DEX were produced and nourished with functional $\mathrm{MHC} /$ peptide complexes capable of promoting $\mathrm{T}$ cell immune responses as well as tumor rejection. By using a process called "direct loading" adopted in a previous study [18], the investigators attached MAGE3 antigenic peptides directly to purified exosomes by incubating them altogether at 10 and $100 \mu \mathrm{g}$ peptide/ml of slightly acidic media. They developed a GMP (Good Manufacturing Practice, a mandatory aspect in pharmaceutical manufacturing) process to produce pharmaceuticalgrade exosomes in large scale. Fifteen stage III/IV melanoma patients received four vaccinations, evaluations were performed before and two weeks after the treatment, no major toxicity (>grade II) was observed. However, the data showed enhanced NK cell effector functions after exosome vaccination in peripheral blood of 8 patients, therefore, they hypothesized that DC derived-exosomes from melanoma patients were specifically endowed with NK cell stimulatory capacity in vivo [19].
Another ongoing clinical trial uses vaccination of recurrent malignant glioma patients. The investigators treat the patients' own tumor cells with an investigational new drug (IGF-1R/AS ODN, an antisense molecule) designed to inactivate targeted surface receptor proteins, and re-implanting the cells after encapsulating them in small bio-diffusion chambers, with the size of a dime, in the patient's abdomen within 24 hours after the craniotomic surgery. As tumor cells die due to apoptosis caused by inactivated surface receptor proteins, they release exosomes containing tumor antigens; working together with the antisense molecules, these antigens trigger immune response against the tumor as they slowly diffuse out of the chamber. Immune cells are readily available outside of the chamber because a wound was created to implant the tumor cells and a foreign object (the chamber) is present. All this would eventually lead to the activation of T cells, which attack and eliminate the tumor. Because this therapy uses a slow-release chamber, and it utilizes the native immune system instead of using artificial engineered cells or biological products, it is considered relatively safer and potentially can generate more benefits with fewer risks [20].

\section{Circulating Tumor Cells}

\section{Advantages of using CTCs}

Circulating Tumor Cells (CTCs) play a significant role in metastasis even though a clear understanding of how they do it is far from complete [21]. CTCs are mostly shed from the primary tumors (i.e. breast cancer, prostate cancer, or brain cancer) during their formation and early growth, and circulate through the bloodstream to potential metastatic sites [22]. Because blood collection is simple and minimally invasive compared to other methods such as needle aspiration of Bone Marrow (BM), CTCs can be used as a real-time marker for disease progression and survival. Moreover, due to their heterogeneity, CTCs can be detected and enriched via different techniques based on their physical properties (i.e. size, density, electric charge) and biological properties (i.e. surface protein expression, invasive capacity), hence, CTC analysis is considered as a real-time "liquid biopsy" in cancer patients [23].

CTCs in cancer patients were first detected in 1869 [24]. Dissemination of tumor cells into the bloodstream from a primary site to a distant organs (i.e. liver, BM, lungs), and the subsequent outgrowth of the tumor in the new microenvironment, can be used as a prognostic biomarker of cancer patients. These cells might stay dormant for years after complete resection of primary tumor before growing into a new tumor; they may also return to the primary site giving rise to a new tumor, a process that is called "local relapse" (Figure 3). Therefore,

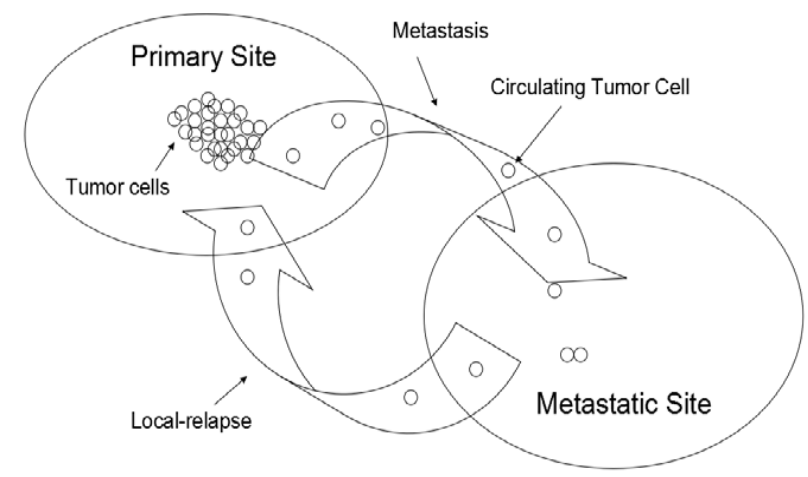

Figure 3: CTC formation. CTCs are shedded from the primary tumors during their formation and early growth, and circulates through the bloodstream to potential metastatic sites. CTCs may stay dormant for years, or undergo "local relapse" - returning to primary site giving rise to a new tumor [25] 
CTCs have the potential to be a promising biomarker for prognosis of very early stage of cancer detection and recurrence of tumors. However, these hypotheses have not been universally proven and further investigation in cancer patients is needed to prove their validity as a surrogate endpoint [25].

\section{Limitations of using CTCs}

The concentration of CTCs in the bloodstream might be as low as one tumor cell in the background of millions of blood cells; thus, identification and characterization of CTCs require extremely sensitive and specific analysis methods, which usually include enrichment and detection procedures based on physical and biological properties [22]. Nonetheless, the site of blood collection for CTC analysis is another critical aspect to consider, because the sizes of CTCs vary at different locations [21]. Some CTCs have a diameter that is three or four times as large as the bores of capillaries, other CTCs may cluster together and lodge in capillaries, and only small and/or plastic CTCs can circulate in bloodstream [26]. Therefore, it is possible that variations exist in the spatial and temporal distribution of CTCs within the circulation.

\section{Isolation and characterization of CTCs}

Isolation of CTCs based on their physical properties has the advantage that they allow CTC enrichment without any labeling. Approaches that have been used to capture CTCs based on physical properties include density gradient centrifugation (Ficoll, OncoQuick), filtration through special filters (Isolation by Size of Epithelial Tumor Cells, or ISET for short); and since cancer cells are larger and stiffer than blood cells, a new versatile label-free biochip is invented for CTC isolation and enrichment. Biological properties are mostly used in immunoselection-based procedures with antibodies against either tumor-associated antigens (positive selection) or the common leukocyte antigen (negative selection) in which mononuclear cells are depleted. Immunomagnetic isolation technique targets an antigen with antibody that is attached to a magnetic bead, and the antigen-antibody complex would then be isolated via exposure to a magnetic field. Antibodies against Epithelial Cell Adhesion Molecule (EpCAM) are usually used as positive selection, and subsequent detection of CTCs is performed with antibodies to cytokeratins; however, this procedure may result in a false-negative finding, because cytokeratins belong to a large family that consists of at least 20 different proteins, and individual cytokeratins (i.e. CK8 or CK18) may be down-regulated in tumor cells. Among the EpCAM-based technologies, the only FDA-approved assay called CellSearch is considered to be the "gold standard" for all new CTC detection methods; however, for cancer patients who lack EpCAM expression, a different antibody, or even method of isolation, is necessary to capture most CTCs in circulation (Figure 4) $[21,22]$.

\section{CTCs in clinical trials}

Several studies have shown that CTCs may be a superior surrogate end point, as they are highly reproducible and correlate better with Overall Survival (OS) than do changes determined by traditional radiology. One such study demonstrated that CTCs are the most accurate and independent predictor of overall survival in CastrationResistant Prostate Cancer (CRPC), and therefore, CTCs can be used in efficacy assessment as surrogate endpoint and expedites the approval of novel anticancer therapies [27]. A total of 276 patients were enrolled in this study and divided into predetermined Favorable $(<5 \mathrm{CTC} / 7.5$ $\mathrm{ml}$ ) and Unfavorable $(>5 \mathrm{CTC} / 7.5 \mathrm{ml})$ groups. Throughout the study, patients within the Unfavorable group at all-time points (71 patients) have the shortest median OS (6.8 months); and patients with increased

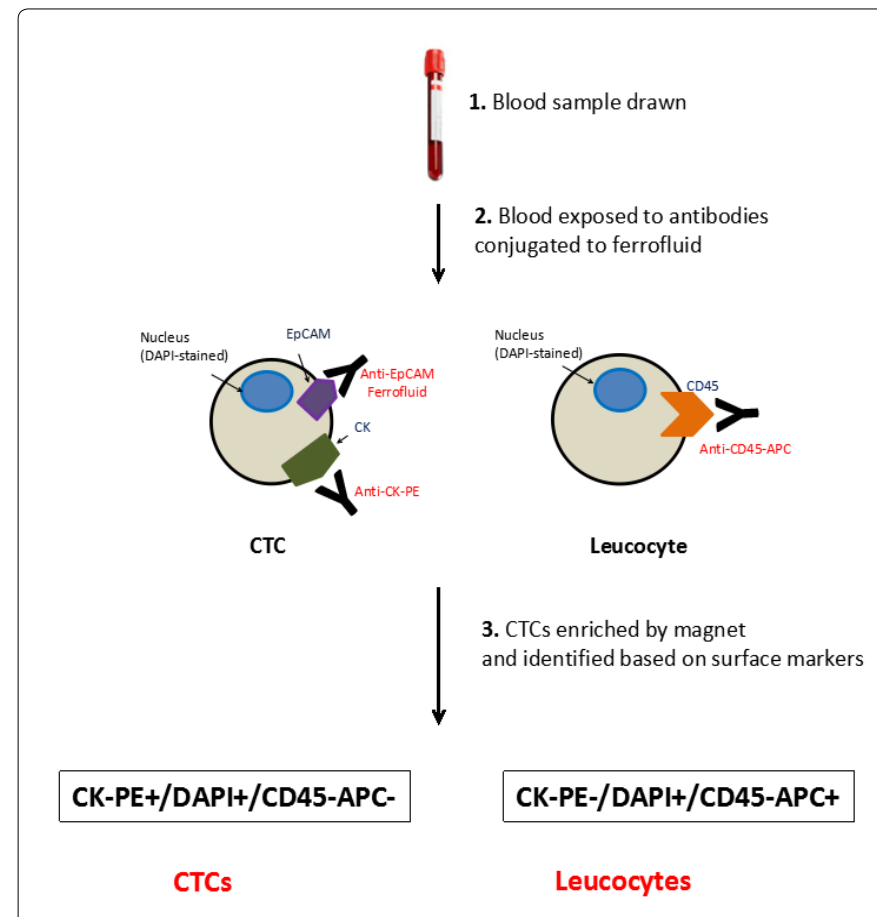

Figure 4: CTC isolation using immunomagnetic selection. A total of 7.5 to $10 \mathrm{~mL}$ of blood is collected and immunomagnetic enrichment is achieved by using the anti-EpCAM (ie, epithelial cell adhesion molecule) Ferrofluid. The isolated cells are then fluorescently labeled with the 4',6-diamidino-2-phenylindole (DAPI) nucleic acid dye and with monoclonal antibodies specific for epithelial cells (ie, anticytokeratin-phycoerythrin to cytokeratins 8/18/19) and leukocytes (ie, anti-CD45-allophycocyanin). CTCs are identified as cells with the appropriate morphology as cytokeratin positive, DAPI positive, and CD45 negative $[21,22]$.

CTC counts after treatment, which converted them from Unfavorable group to Favorable group, showed significant improvement in diagnosis and median OS (21.3 months). In a breast cancer model, there were 177 patients with measurable metastatic breast cancer enrolled, and levels of CTCs were measured both before starting a new line of treatment and at the first follow-up visit. The results showed that patients with CTC levels equal to or higher than 5 per $7.5 \mathrm{ml}$ of whole blood had a shorter median progression-free survival; this difference between groups persisted at the first follow-up visit. Moreover, the proportion of patients with an unfavorable prognosis dropped from $49 \%$ to $30 \%$. The study concluded that CTC level before treatment and at the first follow-up visit is an independent predictor of progression-free survival and overall survival [24].

\section{Circulating Tumor DNA cmDNA as a prognostic tool}

It has been shown that tumor-derived mutant DNA can be detected in the cell-free fraction of the blood of individuals with cancer [2830]. Most of this mutant DNA is not derived from circulating tumor cells and, in light of the specificity of mutations, raises the possibility that the circulating mutant DNA (cmDNA) fragments themselves can be used to track disease status [28-30]. Somatic rearrangements have been shown to occur frequently in cancer. Such alterations are not present in normal cells and should therefore be exquisitely specific and have the potential to serve as highly sensitive biomarkers for tumor detection [31]. Rearrangement-associated biomarkers therefore offer a reliable measure that would be useful for monitoring tumor response to specific therapies. Detection of this circulating mutant DNA in the 
plasma might provide valuable insight on the status of the disease, affect adjuvant treatment decisions, improve early detection of relapse, and enhance patient outcomes.

Indeed, virtually in every cancer cell in a solid tumor, there are approximately 80 genes with subtle mutations, which are not present in normal cells. Therefore in theory, these somatic mutations can serve as highly specific biomarker [31]. It is believed that ctDNA is released into bloodstream when primary tumor cells undergo apoptosis and necrosis processes. ctDNA then circulates predominantly in the form of nucleosomes such that they retain some feature of nuclear chromatin. Besides genetic mutations, epigenetic modifications can also be detected in circulation. Cancer-specific DNA methylation patterns can be found in detached tumor cells in body fluids and biopsies, and they can be detected in free floating DNA that is released from dead cancer cells (Figure 5) [32]. Analysis of DNA methylation test results from cmDNA in Colorectal Cancer (CRC). Currently, evaluation for SEPT9 methylated DNA in peripheral blood is one such marker which has demonstrated feasibility as a blood-based biomarker for all stages and locations of CRC $[33,34]$.

\section{Isolation and quantification of ctDNAs}

In contrast to CTC, ctDNA concentrations in blood are higher in cancer patients than in healthy individuals, yet the number of ctDNA is small compared to the number of normal circulating DNA, representing only a tiny fraction of total circulating DNA (sometimes $<0.01 \%$ ), so it is difficult to detect and quantify it with the sensitivity required for significant clinical use. Nonetheless, this technological challenge can be overcome as new quantification techniques with higher sensitivity are developed. The most commonly accepted quantification procedure is BEAMing (Beads, Emulsification, Amplification, and Magnetics), which analyzes one allele at a time making this method highly sensitive for detection of rare mutant alleles within a large population of wild type alleles, which is the exact microenvironment for ctDNAs. By using this technique, one study concluded that ctDNA measurements can be

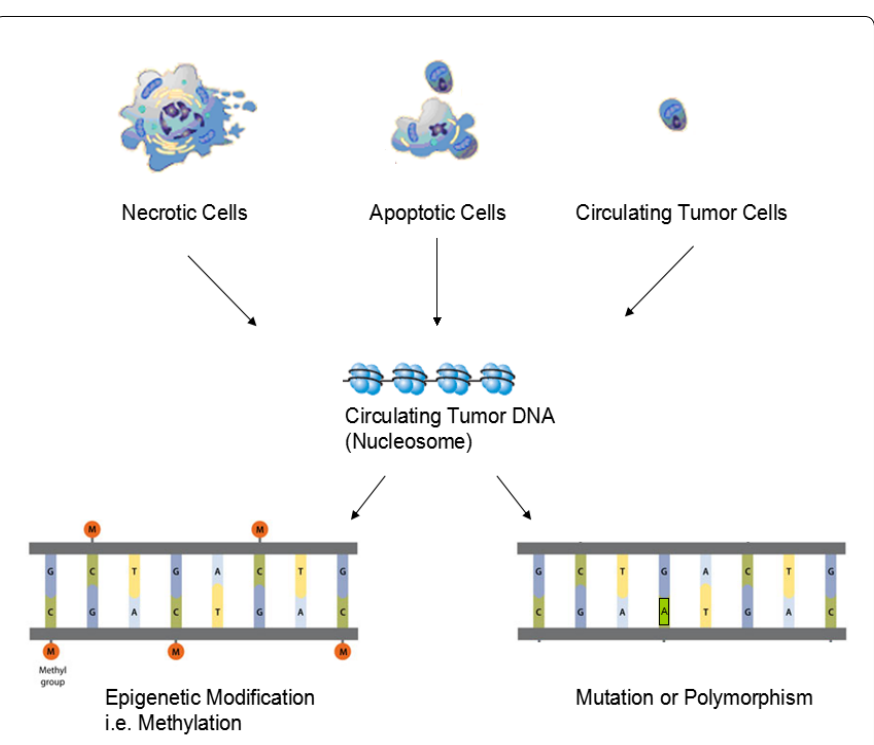

Figure 5: ctDNA formation. Most of ctDNAs are not derived from CTCs, they are released into bloodstream when tumor cells undergo apoptosis or necrosis processes instead. ctDNAs then circulate predominantly in the form of nucleosome to retain some features of nuclear chromatin [28-30]. In addition to genetic mutations, cancer-specific patterns of epigenetic modification, such as methylation, can be found in detached tumor cells in body fluids [32]. used to reliably monitor tumor dynamics in patients with cancer who underwent surgery or chemotherapy. They first assessed 4 genes by direct sequencing in tumors from 18 colorectal cancer patients, and at least one mutation was found in each tumor [31]. After identifying the somatic mutation in each patient's tumor, the median of the estimated total number of DNA fragments was 4000 per millimeter of plasma in 18 patients before surgery. At third and final step, the fraction of DNA fragments of a given gene that contains the queried mutation was determined. They successfully modify the procedure to achieve high signal-to-noise ratio and permit detection of many different mutations via simple hybridization probes under identical conditions. 28 assays were designed for each of the 18 patients, and the median percentage of mutant DNA fragments in 95 positive samples was $0.18 \%$. Multiplying the total number of DNA fragments of a gene in plasma by the fraction of mutant fragments yields the number of ctDNA in plasma. So the median number of ctDNA fragments was 39 in the 95 positive samples.

The first study using BEAMing to detect a drug resistance mutation was published in 2011. Two groups of patients with stage III or IV nonsmall cell lung cancer who had activating EGFR mutations found in diagnostic biopsies. One group had progressive disease after treatment with EGFR-TKI, and a T790M resistance mutation was identified in $43.5 \%$ of patients, a value near the predicted $50 \%$. Another group did not receive the treatment. BEAMing result was reported with sensitivity of $72.7 \%$ in detecting the known activating EGFR mutation in plasma ctDNA collected at a single time point [35]. However, we have used a standard PCR amplification to monitor EGFRvIII deletion in the plasma of glioblastoma patients before and after surgery and have shown that this technique was efficient in predicting the tumor burden in these patients [36].

\section{ctDNA used as biomarker for personalized medicine}

Most established biomarkers for cancers such as Gastrointestinal Stromal Tumor (GIST) possess the limitations of low sensitivity and specificity, and could provide valuable clinical information only in specific settings. CtDNA in theory could use as sensitive, noninvasive biomarker that offers advantages. As tumor-specific biomarker, the amount of ctDNA might be determined by tumor size, vascularization, and cell turnover, which might well reflect disease activity and could be used for monitoring cancer progression. In one study, Jacqueline Maier et al. [37] collected 291 plasma samples from 38 patients with GIST, and developed and validated 25 different allele-specific ligation PCR (L-PCR) assays to detect ctDNA. The data showed that the amount of ctDNA correlated with disease course. Patients with active disease had significantly higher amounts of ctDNA compared with patients with Complete Resection (CR), an increase of ctDNA in patients with tumor progression or relapse was observed, and patients who were responding to treatments (i.e. High-frequency thermo-therapy, imatinib, sunitinib) showed decline of ctDNA. The authors concluded that ctDNA containing tumor-specific mutations could be used as tumor-specific biomarker to predict response early after initiation of treatment; it possesses great potential that might allow earlier treatment changes and avoid repeated tumor biopsies [37-41].

\section{Future Directions and Conclusion}

Early detection of cancer is vital to improved overall survival rates. At present, evidence is accumulating for the clinical value of detecting circulating tumor cells, tumor DNA and microRNA in peripheral blood, plasma, and serum specimens from cancer patients. Both molecular and cellular approaches, which differ in sensitivity and specificity, have been used for such means. Circulating tumor cells and 
extracellular nucleic acids have been detected within blood, plasma, and sera of cancer patients. As the presence of malignant tumors are clinically determined and/or confirmed upon biopsy procurementwhich in itself may have detrimental effects in terms of stimulating cancer progression/metastases-minimally invasive methods would be highly advantageous to the diagnosis and prognosis of cancer and the subsequent tailoring of targeted treatments for individuals, if reliable panels of biomarkers suitable for such an approach exist.

Although, historically, focus has mainly been on proteins and more recently CTCs, results emerging from exploratory studies suggest that cmDNA as well as RNAs contained within exosomes can be amplified using a multiplex approach (thus minimizing the amount of specimen required for analysis) will form members of useful cancer biomarker panels. To date, what could possibly be best described as proof-ofconcept studies have been reported on CTCs, cmDNA and RNAs (mRNAs and miRNAs) as biomarkers. Relative to protein research, however, we believe that this field of research is still in its infancy and should now be much more extensively explored. It is probable that optimal sensitivity and specificity will include a combination of cmDNA, mRNA, miRNA, protein-and possibly also CTC-analysis from a patient's blood specimen. A number of academic laboratories and commercial entities are aware of the huge potential that predictive circulating nucleic acids/CTCs biomarkers represent and their immense clinical importance to the development and improvement of current diagnostic and therapeutic options for cancer patients and are therefore working tirelessly to bring them to clinical practice. Such reliable assays will translate to the clinic and will certainly contribute to improvements in personalized treatment, quality of life and survival for cancer patients.

\section{Acknowledgment}

The authors are supported by funds from the NSF/Mayfield clinic and the University of Cincinnati and the funding sources had no influence on the work cited in this review.

\section{References}

1. Skog J, Würdinger T, van Rijn S, Meijer DH, Gainche L, et al. (2008) Glioblastoma microvesicles transport RNA and proteins that promote tumour growth and provide diagnostic biomarkers. Nat Cell Biol 10: 1470-1476.

2. Azmi AS, Bao B, Sarkar FH (2013) Exosomes in cancer development metastasis, and drug resistance: a comprehensive review. Cancer Metastasis Rev 32: 623-642.

3. Lässer C, Eldh M, Lötvall J (2012) Isolation and characterization of RNAcontaining exosomes. J Vis Exp : e3037.

4. Corrado C, Raimondo S, Chiesi A, Ciccia F, De Leo G, et al. (2013) Exosomes as intercellular signaling organelles involved in health and disease: basic science and clinical applications. Int J Mol Sci 14: 5338-5366.

5. Wieckowski E, Whiteside TL (2006) Human tumor-derived vs dendritic cellderived exosomes have distinct biologic roles and molecular profiles. Immunol Res 36: 247-254.

6. Chaput N, Taïeb J, Schartz N, Flament C, Novault S, et al. (2005) The potentia of exosomes in immunotherapy of cancer. Blood Cells Mol Dis 35: 111-115.

7. Graner MW, Alzate O, Dechkovskaia AM, Keene JD, Sampson JH, et al. (2009) Proteomic and immunologic analyses of brain tumor exosomes. FASEB J 23 . 1541-1557.

8. Vlassov AV, Magdaleno S, Setterquist R, Conrad R (2012) Exosomes: curren knowledge of their composition, biological functions, and diagnostic and therapeutic potentials. Biochim Biophys Acta 1820: 940-948.

9. Yu X, Harris SL, Levine AJ (2006) The regulation of exosome secretion: a nove function of the p53 protein. Cancer Res 66: 4795-4801.

10. Rabinowits G, Gerçel-Taylor C, Day JM, Taylor DD, Kloecker GH (2009) Exosomal microRNA: a diagnostic marker for lung cancer. Clin Lung Cance 10: $42-46$
11. He L, Hannon GJ (2004) MicroRNAs: small RNAs with a big role in gene regulation. Nat Rev Genet 5: 522-531.

12. King HW, Michael MZ, Gleadle JM (2012) Hypoxic enhancement of exosome release by breast cancer cells. BMC Cancer 12: 421.

13. Camacho L, Guerrero P, Marchetti D (2013) MicroRNA and protein profiling of brain metastasis competent cell-derived exosomes. PLoS One 8: e73790.

14. Grange C, Tapparo M, Collino F, Vitillo L, Damasco C, et al. (2011) Microvesicles released from human renal cancer stem cells stimulate angiogenesis and formation of lung premetastatic niche. Cancer Res 71: 5346-5356

15. Hood JL, Pan H, Lanza GM, Wickline SA; Consortium for Translational Research in Advanced Imaging and Nanomedicine (C-TRAIN) (2009) Paracrine induction of endothelium by tumor exosomes. Lab Invest 89: 1317-1328.

16. Hölzel M, Bovier A, Tüting T (2013) Plasticity of tumour and immune cells: a source of heterogeneity and a cause for therapy resistance? Nat Rev Cancer 13: $365-376$

17. Taylor DD, Zacharias W, Gercel-taylor C (2011) Serum/Plasma Proteomics Simpson RJ, Greening DW 728: 235-246.

18. Hsu DH, Paz P, Villaflor G, Rivas A, Mehta-Damani A, et al. (2003) Exosomes as a tumor vaccine: enhancing potency through direct loading of antigenic peptides. J Immunother 26: 440-450.

19. Escudier B, Dorval T, Chaput N, André F, Caby MP, et al. (2005) Vaccination of metastatic melanoma patients with autologous dendritic cell (DC) derivedexosomes: results of thefirst phase I clinical trial. J Transl Med 3: 10.

20. Andrews D (2013) Pilot Immunotherapy Trial for Recurrent Malignant Gliomas

21. Plaks V, Koopman CD, Werb Z (2013) Cancer. Circulating tumor cells. Science 341: 1186-1188.

22. Alix-Panabières $\mathrm{C}$, Schwarzenbach $\mathrm{H}$, Pantel $\mathrm{K}$ (2012) Circulating tumor cells and circulating tumor DNA. Annu Rev Med 63: 199-215.

23. Alix-Panabières C, Pantel K (2013) Circulating tumor cells: liquid biopsy of cancer. Clin Chem 59: 110-118.

24. Cristofanilli M (2006) Circulating tumor cells, disease progression, and survival in metastatic breast cancer. Semin Oncol 33: S9-14.

25. Aguirre-Ghiso JA (2007) Models, mechanisms and clinical evidence for cancer dormancy. Nat Rev Cancer 7: 834-846.

26. Chambers AF, Groom AC, MacDonald IC (2002) Dissemination and growth of cancer cells in metastatic sites. Nat Rev Cancer 2: 563-572.

27. de Bono JS, Scher HI, Montgomery RB, Parker C, Miller MC, et al. (2008) Circulating tumor cells predict survival benefit from treatment in metastatic castration-resistant prostate cancer. Clin Cancer Res 14: 6302-6309.

28. Goebel G, Zitt M, Zitt M, Müller HM (2005) Circulating nucleic acids in plasma or serum (CNAPS) as prognostic and predictive markers in patients with solid neoplasias. Dis Markers 21: 105-120.

29. Fleischhacker M, Schmidt B (2007) Circulating nucleic acids (CNAs) and cancer--a survey. Biochim Biophys Acta 1775: 181-232.

30. Gormally E, Caboux E, Vineis P, Hainaut $P$ (2007) Circulating free DNA in plasma or serum as biomarker of carcinogenesis: practical aspects and biological significance. Mutat Res 635: 105-117.

31. Diehl F, Schmidt K, Choti MA, Romans K, Goodman S, et al. (2008) Circulating mutant DNA to assess tumor dynamics. Nat Med 14: 985-990.

32. Sidransky D (2002) Emerging molecular markers of cancer. Nat Rev Cance 2: 210-219.

33. Warren JD, Xiong W, Bunker AM, Vaughn CP, Furtado LV, et al. (2011) Septin 9 methylated DNA is a sensitive and specific blood test for colorectal cancer. BMC Med 9: 133.

34. Tóth K, Sipos F, Kalmár A, Patai AV, Wichmann B, et al. (2012) Detection of methylated SEPT9 in plasma is a reliable screening method for both left- and right-sided colon cancers. PLoS One 7: e46000.

35. Lauring J, Park BH (2011) BEAMing sheds light on drug resistance. Clin Cancer Res 17: 7508-7510.

36. Salkeni MA, Zarzour A, Ansay TY, McPherson CM, Warnick RE, et al. (2013) Detection of EGFRvIll mutant DNA in the peripheral blood of brain tumo patients. J Neurooncol 115: 27-35. 
Citation: Li Y, Mustapha Bahassi El (2013) Biofluid-Based Circulating Tumor Molecules as Diagnostic Tools for Use in Personalized Medicine. J Mol Biomark Diagn 4: 157. doi:10.4172/2155-9929.1000157

Page 7 of 7

37. Maier J, Lange T, Kerle I, Specht K, Bruegel M, et al. (2013) Detection of mutant free circulating tumor DNA in the plasma of patients with gastrointestinal stromal tumor harboring activating mutations of CKIT or PDGFRA. Clin Cancer Res 19: 4854-4867.

38. Xue X, Teare MD, Holen I, Zhu YM, Woll PJ (2009) Optimizing the yield and utility of circulating cell-free DNA from plasma and serum. Clin Chim Acta 404: 100-104.
39. Clement-Duchene C (2013) Circulating Tumor Cells in Lung cancer (CIRCUBRONCH).

40. Schwartz G Tumor Specific Plasma DNA in Breast Cancer.

41. Mathivanan S, Ji H, Simpson RJ (2010) Exosomes: extracellular organelles important in intercellular communication. J Proteomics 73: 1907-1920.

This article was originally published in a special issue, Biomarkers

Discovery

\& Validation handled by Editor(s). Dr. Krishhan VV, California

State

University, USA 